\title{
MARTINGALE TRANSFORMS WITH UNBOUNDED MULTIPLIERS
}

\author{
J.-A. CHAO AND R.-L. LONG
}

(Communicated by Lawrence F. Gray)

\begin{abstract}
The boundedness of martingale transforms with the "multiplier" sequence in various classes is studied.
\end{abstract}

\section{INTRODUCTION AND PRELIMINARIES}

Let $(X, \mathscr{F}, \mu)$ be a probability space and let $\left\{\mathscr{F}_{n}\right\}_{n \geq 1}$ be a nondecreasing sequence of sub- $\sigma$-fields of $\mathscr{F}$ such that $\mathscr{F}=\mathbf{V} \mathscr{F}_{n}$. We consider processes $f=\{f\}_{n \geq 1}$ adapted to $\left\{\mathscr{F}_{n}\right\}_{n \geq 1}$ and use the convention that $f_{0}=0$. For $0<$ $p \leq \infty$, we say that $f$ is $L^{p}$-bounded and write $f \in L^{p}$ if $\|f\|_{p}=\sup _{n}\left\|f_{n}\right\|_{p}<$ $\infty$. The maximal function of $f$ is defined by $f^{*}=\sup _{n}\left|f_{n}\right|$ and the square function of $f$ is given by $S(f)=\left[\sum_{k=1}^{\infty}\left|d_{k} f\right|^{2}\right]^{1 / 2}$ where $d_{k} f=f_{k}-f_{k-1}$, $k=1,2, \cdots$, is the difference sequence of $f$. For martingales, we consider the following versions of Hardy spaces, $0<p \leq \infty$ :

$$
\begin{aligned}
& H_{*}^{p}=\left\{f:\|f\|_{H_{*}^{p}}=\left\|f^{*}\right\|_{p}<\infty\right\} ; \\
& H_{S}^{p}=\left\{f:\|f\|_{H_{S}^{p}}=\|S(f)\|_{p}<\infty\right\} .
\end{aligned}
$$

It is well known that $H_{*}^{p} \approx L^{p} \approx H_{S}^{p}$ for $1<p<\infty$, and Davis [8] proved that $H_{*}^{1} \approx H_{S}^{1}$. Note that, in general, $H_{*}^{p} \not H_{S}^{p}$ when $0<p<1$. We shall denote $H^{p} \equiv H_{*}^{p} \approx H_{S}^{p}$ whenever $1 \leq p<\infty$. We also consider the conditioned square function of $f$ given by

$$
s(f)=\left[\sum_{k=1}^{\infty} E\left(\left|d_{k} f\right|^{2} \mid \mathscr{F}_{k-1}\right)\right]^{1 / 2},
$$

Received by the editors July 6, 1990; preliminary versions of the paper were presented at the Conference on Operators on Martingales, University of Arkansas at Fayetteville, April 1987 and in the Special Session on Harmonic Analysis and Probability at the American Mathematical Society Meeting in Manhatten, Kansas, March 1990.

1980 Mathematics Subject Classification (1985 Revision). Primary 60G46, 42B30; Secondary $60 \mathrm{G} 42$.

Key words and phrases. Martingale transform, Hardy spaces, maximal function, square functions, BMO, extrapolation.

Research partially supported by grants from Cleveland State University and National Science Foundation of China. 
and the Hardy space it defines:

$$
\mathbf{h}^{p}=\left\{f:\|f\|_{\mathbf{h}^{p}}=\|s(f)\|_{p}<\infty\right\} .
$$

This version of Hardy spaces is more interesting to study when $0<p \leq 1$. The spaces of martingales with bounded mean oscillation are defined, for $1 \leq p<$ $\infty$, by

$$
\begin{aligned}
\mathbf{B M O}_{p} & =\left\{f:\|f\|_{\mathbf{B M O}_{p}}=\sup _{n}\left\|\left[E\left(\left|\sum_{k=n}^{\infty} d_{k} f\right|^{p} \mid \mathscr{F}_{n}\right)\right]^{1 / p}\right\|_{\infty}<\infty\right\} ; \\
\mathbf{b m o}_{p} & =\left\{f:\|f\|_{\text {bmo }_{p}}=\sup _{n}\left\|\left[E\left(\left|\sum_{k=n+1}^{\infty} d_{k} f\right|^{p} \mid \mathscr{F}_{n}\right)\right]^{1 / p}\right\|_{\infty}<\infty\right\} .
\end{aligned}
$$

The John-Nirenberg theorem gives that all $\mathbf{B M O}_{p}$ spaces are equivalent for $1 \leq p<\infty$. We shall denote them simply by BMO and the norm by $\|\cdot\|_{*}$. However, bmo $_{p}$ form a decreasing family as the index $p$ increases. Among them, the most important ones are $\mathbf{b m o}_{1}$ and $\mathbf{b m o}_{2}$. Fefferman's duality theorem says that BMO is the dual space of $H^{1}$, and Herz [10] showed that $\mathbf{b m o}_{2}$ is the dual space of $\mathbf{h}^{1}$. Moreover, Herz [10] obtained that the dual spaces of $H_{S}^{p}$ and $\mathbf{h}^{p}$ are $\Lambda_{\alpha}$ and $\lambda_{\alpha}$, respectively, where $\alpha=\frac{1}{p}-1 \geq 0$, and

$$
\begin{aligned}
\Lambda_{\alpha} & =\left\{f \in L^{2}:\|f\|_{\Lambda_{\alpha}}=\sup _{n}\left\|\omega_{n}^{-\alpha} E\left(\left|\sum_{k=n}^{\infty} d_{k} f\right|^{2} \mid \mathscr{F}_{n}\right)^{1 / 2}\right\|_{\infty}<\infty\right\} ; \\
\lambda_{\alpha} & =\left\{f \in L^{2}:\|f\|_{\lambda_{\alpha}}=\sup _{n}\left\|\omega_{n}^{-\alpha} E\left(\left|\sum_{k=n+1}^{\infty} d_{k} f\right|^{2} \mid \mathscr{F}_{n}\right)^{1 / 2}\right\|_{\infty}<\infty\right\},
\end{aligned}
$$

with $\omega_{n}=\sum|I| \chi_{I}$; here the summation is over all $\mathscr{F}_{n}$-atoms $I$ and $|I|$ denotes the measure of $I$. Note that $\Lambda_{0}=\mathbf{B M O}$ and $\lambda_{0}=\mathbf{b m o}_{2}$.

We introduce the following classes of processes $v=\left\{v_{n}\right\}_{n \geq 1}$ adapted to $\left\{\mathscr{F}_{n}\right\}_{n \geq 1}$ :

$$
V^{p}=\left\{v:\|v\|_{V^{p}}=\left\|v^{*}\right\|_{p}<\infty\right\}, \quad 0<p \leq \infty .
$$

The martingale transform $T_{v}$ for a given $v$ is defined by $T_{v} f=\sum_{n=1}^{\infty} v_{n-1} d_{n} f$. Burkholder [3] showed that when $v \in V^{\infty}$, then $T_{v}$ is of type $(p, p)$ for $1<$ $p<\infty$ and of weak type $(1,1)$. The purpose of this paper is to study certain boundedness behaviors of the transform $T_{v}$ when the "multiplier" sequence $v$ lies in other $V^{p}$ classes. A special case of our main result is that, for $v \in V^{p}$ with $0<p<\infty, T_{v}$ is bounded from BMO to the Hardy spaces $H_{*}^{p}$ and $H_{S}^{p}$. A similar result in the continuous parameter case for Brownian martingales was obtained recently by Bañuelos and Bennett [1]. However, when $0<p \leq 1$, their argument depends on the atomic decomposition, which is not available for the discrete case in general. The main idea in our proof for $0<p \leq 1$ involves a certain commutability concept that is used to obtain the extrapolated results. The method of extrapolation was first introduced by Burkholder and Gundy in [5].

\section{BOUNDEDNESS OF MARTINGALE TRANSFORMS}

We first present two elementary results on the boundedness of $T_{v}$. 
Theorem 1. If $0<p, q \leq \infty, v \in V^{p}$ and $\frac{1}{r}=\frac{1}{p}+\frac{1}{q}$, then $T_{v}$ is of types $\left(H_{S}^{q}, H_{S}^{r}\right)$ and $\left(\mathbf{h}^{q}, \mathbf{h}^{r}\right)$ with $\left\|T_{v}\right\| \leq\|v\|_{V^{p}}$.

Proof. This follows directly from the pointwise estimates:

$$
\begin{gathered}
S\left(T_{v} f\right)(x) \leq v^{*}(x) S(f)(x) \quad \text { a.e. } \\
s\left(T_{v} f\right)(x) \leq v^{*}(x) s(f)(x) \quad \text { a.e. }
\end{gathered}
$$

A similar result for stochastic integrals was obtained by Bichteler [2].

Note that $T_{v}$ is selfadjoint in the sense that for nice $f$ and $g, E\left(g T_{v} f\right)$ $=E\left(f T_{v} g\right)$. Using this and a duality argument, we obtain

Theorem 2. Let $0 \leq \alpha<\infty, \frac{1}{1+\alpha}<p \leq \infty$ and $v \in V^{p}$. Then

(i) $T_{v}$ is of types $\left(\Lambda_{\alpha}, \Lambda_{\beta}\right)$ and $\left(\boldsymbol{\lambda}_{\alpha}, \boldsymbol{\lambda}_{\beta}\right)$ where $\beta=\alpha-\frac{1}{p} \geq 0$ (i.e., $\left.\frac{1}{\alpha} \leq p \leq \infty\right)$;

(ii) $T_{v}$ is of types $\left(\Lambda_{\alpha}, H^{r}\right)$ and $\left(\boldsymbol{\lambda}_{\alpha}, \mathbf{h}^{r}\right)$ where $0<\frac{1}{r}=\frac{1}{p}-\alpha<1$ (i.e., $\frac{1}{1+\alpha}<p<\frac{1}{\alpha}$ ).

In both cases, $\left\|T_{v}\right\| \leq C\|v\|_{V^{p}}$.

Proof. Set $t=\frac{1}{1+\alpha}$. When $\frac{1}{p} \leq \alpha$, let $q(0<q \leq 1)$ be such that $\frac{1}{p}+\frac{1}{q}=$ $1+\alpha=\frac{1}{t}$. Note that $\alpha=\frac{1}{t}-1$ and $\beta=\frac{1}{q}-1$. For nice $f \in \Lambda_{\alpha}$ and $g \in H_{S}^{q}$, from Theorem 1 and Herz's duality result [10], we have

$$
\left|E\left(g T_{v} f\right)\right|=\left|E\left(f T_{v} g\right)\right| \leq C\|f\|_{\Lambda_{\alpha}}\left\|T_{v} g\right\|_{H_{S}^{t}} \leq C\|v\|_{V^{p}}\|f\|_{\Lambda_{\alpha}}\|g\|_{H_{S}^{q}} .
$$

Hence $T_{v} f \in\left(H_{S}^{q}\right)^{\prime}=\Lambda_{\beta}$ and $\left\|T_{v} f\right\|_{\Lambda_{\beta}} \leq C\|v\|_{V^{p}}\|f\|_{\Lambda_{\alpha}}$.

When $\alpha<\frac{1}{p}<1+\alpha$, let $q(1<q<\infty)$ be such that $\frac{1}{p}+\frac{1}{q}=\frac{1}{t}$. Note that in this case, $\frac{1}{q}+\frac{1}{r}=1$. The same duality argument shows that $T_{v} f \in\left(H_{S}^{q}\right)^{\prime}=H^{r}$ and $\left\|T_{v} f\right\|_{r} \leq C\|v\|_{V^{p}}\|f\|_{\Lambda_{\alpha}}$.

The remaining statements involving $\lambda_{\alpha}$ follow from a similar argument using the duality results in [10].

We single out a very special case of Theorem 2(ii) when $\alpha=0$ :

Corollary 3. For $f \in \mathbf{B M O}$ and $v \in V^{p}$ with $1<p<\infty, T_{v} f \in L^{p}$ and $\left\|T_{v} f\right\|_{p} \leq C\|v\|_{V^{p}}\|f\|_{*}$.

We note here that, for martingales $v$ and $f, T(v, f)=\sum_{n} v_{n-1} d_{n} f$ is a martingale version of the paraproduct as studied in Coifman-Meyer [6, 7]. Corollary 3 corresponds to a fundamental result on paraproducts in the study of pseudodifferential operators. (See [6].) A similar result for stochastic integrals was obtained by Lepingle [11].

We shall use the idea of extrapolation to treat the case not covered by Theorem 2, i.e., $p \leq \frac{1}{1+\alpha}$. We introduce the following notion of commutability:

Definition. A martingale valued linear operator $T$ defined on $V^{\infty}$ is $*$-quasicommutable with stopping times if, for all stopping times $\tau$ and $v \in V^{\infty}$,

$$
\left(T\left(v-v^{(\tau-1)}\right)\right)^{*} \chi_{\{\tau=\infty\}}=0 \text { a.e. }
$$


$T$ is $S$-quasicommutable with stopping times if, for all stopping times $\tau$ and $v \in V^{\infty}$,

$$
S\left(T\left(v-v^{(\tau-1)}\right)\right) \chi_{\{\tau=\infty\}}=0 \text { a.e.; }
$$

$T$ is $s$-quasicommutable with stopping times if, for all stoppings time $\tau$ and $v \in V^{\infty}$,

$$
s\left(T\left(v-v^{(\tau-1)}\right)\right) \chi_{\{\tau=\infty\}}=0 \text { a.e., }
$$

where the process $v^{(\tau-1)}$ is given as usual by

$$
\begin{gathered}
v^{(\tau-1)}=\left\{v_{(\tau-1) \wedge n}\right\}_{n \geq 1} \\
\text { with } v_{(\tau-1) \wedge n}=v_{1} \chi_{\{\tau=2\}}+\cdots+v_{n} \chi_{\{\tau \geq n+1\}}, \quad \text { for } n \geq 1 .
\end{gathered}
$$

Lemma 4. Let $0<p_{0} \leq r_{0} \leq \infty$ and $T$ be a martingale valued linear operator on $v \in V^{\infty}$.

(i) If $T$ is *-quasicommutable with stopping times and is of the weak type $\left(V^{p_{0}}, H_{*}^{r_{0}}\right)$ with the bound $\|T\|$, then for all pairs $(p, r)$ satisfying

$$
\frac{1}{p}-\frac{1}{r}=\frac{1}{p_{0}}-\frac{1}{r_{0}}, \quad 0<p<p_{0},
$$

$T$ is of type $\left(V^{p}, H_{*}^{r}\right)$ with the bound $C\|T\|$.

(ii) If $T$ is $S$-quasicommutable with stopping times and is of weak type $\left(V^{p_{0}}, H_{S}^{r_{0}}\right)$ with the bound $\|T\|$, then for all pairs $(p, r)$ satisfying (4), $T$ is of type $\left(V^{p}, H_{S}^{r}\right)$ with the bound $C\|T\|$.

(iii) If $T$ is s-quasicommutable with stopping times and is of weak type $\left(V^{p_{0}}, \mathbf{h}^{r_{0}}\right)$ with the bound $\|T\|$, then for all pairs $(p, r)$ satisfying (4), $T$ is of type $\left(V^{p}, \mathbf{h}^{r}\right)$ with the bound $C\|T\|$.

Proof. (i) Suppose $T$ is *-quasicommutable with stopping times and is of weak type $\left(V^{p_{0}}, H_{*}^{r_{0}}\right)$. We first assume that $\|T\|=1$ and $\|v\|_{V^{p}}=1$ for a given $v \in V^{\infty}$. For $\lambda>0$, set $\delta=\lambda^{r / p}$, and consider the stopping time $\tau=\inf \left\{n:\left|v_{n}\right|>\delta\right\}$. We have $\{\tau<\infty\}=\left\{v^{*}>\delta\right\}$ and $\left(v^{(\tau-1)}\right)^{*} \leq \delta$. Write $v=v-v^{(\tau-1)}+v^{(\tau-1)}$. Using (1), we get

$$
\begin{aligned}
\left\{(T(v))^{*}>2 \lambda\right\} & \subset\left\{\left(T\left(v-v^{(\tau-1)}\right)\right)^{*}>\lambda\right\} \cup\left\{\left(T\left(v^{(\tau-1)}\right)\right)^{*}>\lambda\right\} \\
& \subset\{\tau<\infty\} \cup\left\{\left(T\left(v^{(\tau-1)}\right)\right)^{*}>\lambda\right\} .
\end{aligned}
$$

It follows from the weak type property of $T$ that

$$
\begin{aligned}
\left|\left\{(T(v))^{*}>2 \lambda\right\}\right| & \leq|\{\tau<\infty\}|+\frac{C}{\lambda^{r_{0}}}\left[\int_{\{\tau<\infty\}}+\int_{\{\tau=\infty\}}\left(v^{(\tau-1)}\right)^{* p_{0}} d \mu\right]^{r_{0} / p_{0}} \\
& \leq|\{\tau<\infty\}|+\frac{C \delta^{r_{0}}}{\lambda^{r_{0}}}|\{\tau<\infty\}|^{r_{0} / p_{0}}+\frac{C}{\lambda^{r_{0}}}\left[\int_{\left\{v^{*} \leq \delta\right\}} v^{* p_{0}} d \mu\right]^{r_{0} / p_{0}} \\
& =I_{1}+I_{2}+I_{3}, \quad \text { say. }
\end{aligned}
$$


Now

$$
\begin{aligned}
\int_{0}^{\infty} \lambda^{r-1} I_{1} d \lambda & =\int_{0}^{\infty} \lambda^{r-1}\left|\left\{v^{*}>\delta=\lambda^{r / p}\right\}\right| d \lambda \\
& =C \int_{0}^{\infty} t^{p-1}\left|\left\{v^{*}>t\right\}\right| d t=C\left\|v^{*}\right\|_{p}^{p}=C .
\end{aligned}
$$

We note that

$$
\begin{aligned}
\frac{\delta^{r_{0}}}{\lambda^{r_{0}}}|\{\tau<\infty\}|^{r_{0} / p_{0}-1} & =\frac{\delta^{r_{0}-p\left(r_{0} / p_{0}-1\right)}}{\lambda^{r_{0}}}\left[\delta^{p}\left|\left\{v^{*}>\delta\right\}\right|\right]^{r_{0} / p_{0}-1} \\
& \leq \lambda^{(r / p)\left(r_{0}-p\left(r_{0} / p_{0}-1\right)\right)-r_{0}}\left[\int_{\Omega} v^{* p} d \mu\right]^{r_{0} / p_{0}-1} \\
& =\lambda^{r r_{0}\left(1 / p-1 / p_{0}+1 / r_{0}\right)-r_{0}}\left\|v^{*}\right\|_{p}^{p\left(r_{0} / p_{0}-1\right)}=1
\end{aligned}
$$

because of (4). Thus

$$
\int_{0}^{\infty} \lambda^{r-1} I_{2} d \lambda \leq C \int_{0}^{\infty} \lambda^{r-1}|\{\tau<\infty\}| d \lambda \leq C .
$$

For an estimate involving $I_{3}$, denoting $a=\frac{r\left(p-p_{0}\right)}{p}-1<-1$, we have

$$
\begin{aligned}
\int_{0}^{\infty} & \lambda^{r-1} I_{3} d \lambda=C \int_{0}^{\infty} \lambda^{r-1-r_{0}-a} \lambda^{a}\left(\int_{\left\{v^{*} \leq \delta\right\}} v^{* p_{0}} d \mu\right)^{r_{0} / p_{0}} d \lambda \\
& \leq C \sup _{\lambda}\left\{\lambda^{r-1-r_{0}-a}\left(\int_{\left\{v^{*} \leq \delta\right\}} v^{* p_{0}} d \mu\right)^{r_{0} / p_{0}-1}\right\} \cdot \int_{0}^{\infty} \lambda^{a} \int_{\left\{v^{*} \leq \delta\right\}} v^{* p_{0}} d \mu d \lambda \\
& =C J_{1} \cdot J_{2}, \quad \text { say. }
\end{aligned}
$$

Here

$$
\begin{aligned}
J_{1} & \leq \sup _{\lambda}\left\{\lambda^{r-1-r_{0}-a} \delta^{\left(p_{0}-p\right)\left(r_{0} / p_{0}-1\right)}\right\}\left\|v^{*}\right\|_{p}^{p\left(r_{0} / p_{0}-1\right)} \\
& =\sup _{\lambda}\left\{\lambda^{r-r_{0}+\left(r r_{0} / p p_{0}\right)\left(p_{0}-p\right)}\right\} \leq 1 ; \\
J_{2} & =\int_{\Omega} \int_{v^{* p / r}}^{\infty} \lambda^{a} d \lambda v^{* p_{0}} d \mu \leq C \int_{\Omega} v^{*(p / r)(a+1)+p_{0}} d \mu=C \int_{\Omega} v^{* p} d \mu=C .
\end{aligned}
$$

Combining these estimates, we get, with $\|T\|=1$,

$$
\int_{0}^{\infty} \lambda^{r-1}\left|\left\{(T(v))^{*}>2 \lambda\right\}\right| d \lambda \leq C \text { for all } v \in V^{\infty} \text { with }\|v\|_{V^{p}}=1 .
$$

Since $V^{\infty}$ is dense in $V^{p}$, this inequality is valid for all $v \in V^{p}$.

Therefore from the linearity, we have, in general,

$$
\|T(v)\|_{H^{r}} \leq C\|T\|\|v\|_{V^{p}} \text { for all } v \in V^{p} .
$$

This completes the proof of (i).

The proofs of parts (ii) and (iii) are similar. For instance, if $T$ is $S$ quasicommutable with stopping times and is of weak type $\left(V^{p_{0}}, H_{S}^{r_{0}}\right)$, then, 
with the same stopping time $\tau$,

$$
\begin{aligned}
\{S(T(v))>2 \lambda\} & \subset\left\{S\left(T\left(v-v^{(\tau-1)}\right)\right)>\lambda\right\} \cup\left\{S\left(T\left(v^{(\tau-1)}\right)\right)>\lambda\right\} \\
& \subset\{\tau<\infty\} \cup\left\{S\left(T\left(v^{(\tau-1)}\right)\right)>\lambda\right\},
\end{aligned}
$$

and

$$
|\{S(T(v))>2 \lambda\}| \leq|\{\tau<\infty\}|+\frac{C}{\lambda^{r_{0}}}\left[\int_{\{\tau<\infty\}}+\int_{\{\tau=\infty\}}\left(v^{(\tau-1)}\right)^{* p_{0}} d \mu\right]^{r_{0} / p_{0}} .
$$

The rest of the proof follows the estimates for (i). The same is true for the proof of (iii) concerning the conditioned square functions $s$.

Our main result is the following extension of Theorem 2 for the case $0<$ $p<\frac{1}{\alpha}$.

Theorem 5. Let $0 \leq \alpha<\infty, 0<p<\frac{1}{\alpha}$ and $v \in V^{p}$, and set $\frac{1}{r}=\frac{1}{p}-\alpha$. Then $T_{v}$ is of types $\left(\Lambda_{\alpha}, H_{*}^{r}\right),\left(\Lambda_{\alpha}, H_{S}^{r}\right)$ and $\left(\boldsymbol{\lambda}_{\alpha}, \mathbf{h}^{r}\right)$ with the bound $C\|v\|_{V^{p}}$.

Proof. The case $\frac{1}{1+\alpha}<p<\frac{1}{\alpha}$ has been covered by Theorem 2(ii). Assume that $p \leq \frac{1}{1+\alpha}$. Let $f \in \Lambda_{\alpha}$ (or $\lambda_{\alpha}$ ) be given and fixed. Consider $T_{v} f$ as an operator $T$ defined on $V^{\infty}$. From Theorem 2(ii), we know that $T$ (for the fixed $f$ ) is of type $\left(V^{p_{0}}, H^{r_{0}}\right)$ (or $\left.\left(V^{p_{0}}, \mathbf{h}^{r_{0}}\right)\right)$, for some $\left(p_{0}, r_{0}\right)$ such that $\frac{1}{1+\alpha}<p_{0}<\frac{1}{\alpha}$ and $\frac{1}{r_{0}}=\frac{1}{p_{0}}-\alpha<1$ with the bound $C\|f\|_{\Lambda_{\alpha}}$ (or $C\|f\|_{\lambda_{\alpha}}$ ). From Lemma 4, the desired boundedness properties follow provided that $T$ satisfies the various quasicommutabilities with stopping times.

Let $v \in V^{\infty}$ and $\tau$ be any stopping time. Since

$$
\left(v-v^{(\tau-1)}\right)_{k-1}=v_{k-1}-\left(v_{1} \chi_{\{\tau=2\}}+\cdots+v_{k-1} \chi_{\{\tau \geq k\}}\right), \quad k \geq 1,
$$

we have

$$
\begin{aligned}
\left(T_{v-v^{(\tau-1)}} f\right)_{n} & =\sum_{k=1}^{n}\left(v-v^{(\tau-1)}\right)_{k-1} d_{k} f, \quad n \geq 1 ; \\
S_{n}\left(T_{v-v^{(\tau-1)}} f\right) & =\left[\sum_{k=1}^{n}\left|\left(v-v^{(\tau-1)}\right)_{k-1}\right|^{2}\left|d_{k} f\right|^{2}\right]^{1 / 2}, \quad n \geq 1 ; \\
s_{n}\left(T_{v-v^{(\tau-1)}} f\right) & =\left[\sum_{k=1}^{n}\left|\left(v-v^{(\tau-1)}\right)_{k-1}\right|^{2} E\left(\left|d_{k} f\right|^{2} \mid \mathscr{F}_{k-1}\right)\right]^{1 / 2}, \quad n \geq 1 .
\end{aligned}
$$

They all vanish on the set $\{\tau=\infty\}$. Therefore the proof of Theorem 5 is completed.

Again, we single out the special case when $\alpha=0$.

Corollary 6. For $0<p<\infty$ and $v \in V^{p}$,

$$
\begin{array}{lr}
\left\|T_{v} f\right\|_{H^{p}} \leq C\|v\|_{V^{p}}\|f\|_{*}, & f \in \mathbf{B M O} ; \\
\left\|T_{v} f\right\|_{H_{S}^{p}} \leq C\|v\|_{V^{p}}\|f\|_{*}, & f \in \mathbf{B M O} \\
\left\|T_{v} f\right\|_{\mathbf{h}^{p}} \leq C\|c\|_{V^{p}}\|f\|_{\text {bmo }_{2}}, & f \in \text { bmo }_{2} .
\end{array}
$$


The boundedness behaviors of the transform $T_{v}$ on $H_{S}^{q}, \mathbf{h}^{q}$ and $\Lambda_{\alpha}, \lambda_{\alpha}$ have been satisfactory, but not on maximal Hardy spaces $H_{*}^{q}$. For instance, the property parallel to Theorem 1 for $H_{*}^{q}$ is not readily obtainable. Nevertheless, we have the following boundedness result of $T_{v}$ on $H_{*}^{q}$ with the restriction $q \geq 1$.

Theorem 7. Let $0<p \leq \infty, 1 \leq q<\infty, \frac{1}{r}=\frac{1}{p}+\frac{1}{q}$ and $v \in V^{p}$. Then $T_{v}$ is of type $\left(H^{q}, H_{*}^{r}\right)$ with the bound $C\|v\|_{V^{p}}$.

Proof. From Davis's decomposition [8] for $f \in H^{q}, q \geq 1$, we have $f=$ $f^{(1)}+f^{(2)}$ with

$$
\begin{gathered}
\left\|\sum\left|d_{n} f^{(1)}\right|\right\|_{q} \leq C\|f\|_{H^{q}} ; \\
\left|d_{n} f^{(2)}\right| \leq C f_{n-1}^{*} \text { and }\left\|f^{(2)}\right\|_{H^{q}} \leq C\|f\|_{H^{q}} .
\end{gathered}
$$

(This decomposition for the case $q>1$ is obtained similarly as for $q=1$ in [8].) Hence,

$$
\begin{aligned}
\left\|\left(T_{v} f\right)^{*}\right\|_{r} & \leq C\left(\left\|\left(T_{v} f^{(1)}\right)^{*}\right\|_{r}+\left\|\left(T_{v} f^{(2)}\right)^{*}\right\|_{r}\right) \\
& \leq C\|v\|_{V^{p}}\left(\left\|\sum\left|d_{n} f^{(1)}\right|\right\|_{q}+\left\|f^{(2)}\right\|_{H^{q}}\right) \leq C\|v\|_{V^{p}}\|f\|_{H^{q}} .
\end{aligned}
$$

Theorem 7 does not cover the case that $q=\infty$. In this case, the spaces $H_{*}^{q}$ and $H_{S}^{q}$ should be replaced by BMO for the corresponding results as obtained in Corollary 6. For the case when $p=\infty$ and $q=\infty$, we have that $T_{v}$, with $v \in V^{\infty}$, is of type (BMO, BMO) as a special case of Theorem 2(i).

We finish this section by providing the weak type boundedness of $T_{v}$ on $L^{1}$, as one would expect.

Theorem 8. Let $0<p \leq \infty, v \in V^{p}$ and $\frac{1}{r_{0}}=\frac{1}{p}+1$. Then $T_{v}$ is of weak types $\left(L^{1}, H_{*}^{r_{0}}\right)$ and $\left(L^{1}, H_{S}^{r_{0}}\right)$. Namely, for all $L^{1}$-bounded martingale $f, \lambda>0$,

$$
\left|\left\{\left(T_{v} f\right)^{*}>\lambda\right\}\right| \leq\left(\frac{C}{\lambda}\|f\|_{1}\right)^{r_{0}} ; \quad\left|\left\{S\left(T_{v} f\right)>\lambda\right\}\right| \leq\left(\frac{C}{\lambda}\|f\|_{1}\right)^{r_{0}} .
$$

Proof. Without loss of generality, we assume $\|v\|_{V^{p}}=\|f\|_{1}=1$. Do a Gundy decomposition [9] on $f$ with $\delta=\lambda^{r_{0}}$, we have $f=f^{(1)}+f^{(2)}+f^{(3)}$ with

$$
\begin{gathered}
\left\|f^{(1)}\right\|_{1} \leq C \quad \text { and }|A|=\left|\left\{\sup _{n}\left|d_{n} f^{(1)}\right| \neq 0\right\}\right| \leq \frac{C}{\delta} ; \\
\qquad \sum\left|d_{n} f^{(2)}\right| \|_{1} \leq C, \\
\left\|f^{(3)}\right\|_{\infty} \leq C \delta \text { and }\left\|f^{(3)}\right\|_{p}^{p} \leq C \delta^{p-1}, \quad 1 \leq p<\infty .
\end{gathered}
$$

Since

$$
\left\{\left(T_{v} f\right)^{*}>2 \lambda\right\} \subset\left\{\left(T_{v} f^{(1)}\right)^{*} \neq 0\right\} \cup\left\{\left(T_{v} f^{(2)}\right)^{*}>\lambda\right\} \cup\left\{\left(T_{v} f^{(3)}\right)^{*}>\lambda\right\},
$$

we get, letting $r$ be such that $\frac{1}{r}=\frac{1}{p}+\frac{1}{2}$,

$$
\begin{aligned}
\mid\left\{\left(T_{v} f\right)^{*}\right. & >2 \lambda\}|\leq| A \mid+\lambda^{-r_{0}}\left\|\left(T_{v} f^{(2)}\right)^{*}\right\|_{r_{0}}^{r_{0}}+C \lambda^{-r}\left\|f^{(3)}\right\|_{2}^{r} \\
& \leq C \delta^{-1}+\lambda^{-r_{0}}\left\|v^{*} \sum\left|d_{n} f^{(2)}\right|\right\|_{r_{0}}^{r_{0}}+C \lambda^{-r} \delta^{r / 2} \\
& \leq C \lambda^{-r_{0}}+\lambda^{-r_{0}}\left(\left\|v^{*}\right\|_{p}\left\|\sum\left|d_{n} f^{(2)}\right|\right\|_{1}\right)^{r_{0}}+C \lambda^{r r_{0}\left(1 / 2-1 / r_{0}\right)} \leq C \lambda^{-r_{0}} .
\end{aligned}
$$


A similar argument gives $\left|\left\{S\left(T_{v} f\right)>2 \lambda\right\}\right| \leq C \lambda^{-r_{0}}$. Linearity completes the proof of Theorem 8 .

As a summary, we list the results obtained in this section by treating the martingale transform as a bilinear operator $T:(v, f) \rightarrow T_{v} f . T$ is then of the following types, $0<p \leq \infty$ :

$$
\begin{aligned}
& \left(V^{p}, H_{S}^{q} ; H_{S}^{r}\right) \quad \text { and }\left(V^{p}, \mathbf{h}^{q} ; \mathbf{h}^{r}\right), \quad 0<q \leq \infty, \frac{1}{r}=\frac{1}{p}+\frac{1}{q} ; \\
& \left(V^{p}, \Lambda_{\alpha} ; \Lambda_{\beta}\right) \text { and }\left(V^{p}, \lambda_{\alpha} ; \lambda_{\beta}\right), \quad 0 \leq \alpha<\infty, \beta=\alpha-\frac{1}{p} \geq 0 ; \\
& \left(V^{p}, \Lambda_{\alpha} ; H_{*}^{r}\right),\left(V^{p}, \Lambda_{\alpha} ; H_{S}^{r}\right) \quad \text { and } \quad\left(V^{p}, \lambda_{\alpha} ; \mathbf{h}^{r}\right), \\
& \quad \frac{1}{r}=\frac{1}{p}-\alpha>0, \quad\left(0<p<\frac{1}{\alpha}\right) ; \\
& \left(V^{p}, H^{q} ; H_{*}^{r}\right), \quad 1 \leq q<\infty, \frac{1}{r}=\frac{1}{p}+\frac{1}{q} ; \\
& \left(V^{p}, L^{1} ; \mathrm{w} H_{*}^{r_{0}}\right) \quad \text { and }\left(V^{p}, L^{1} ; \mathrm{w} H_{S}^{r_{0}}\right), \quad \frac{1}{r_{0}}=\frac{1}{p}+1 .
\end{aligned}
$$

When both $v$ and $f$ are martingales, $T$ is one version of paraproducts on martingales. The properties of various variants of paraproducts on martingales and certain necessary conditions for the boundedness of these transforms will be discussed in a sequel to this paper.

\section{REFERENCES}

1. R. Bañuelos and A. G. Bennett, Paraproducts and commutators of martingale transforms, Proc. Amer. Math. Soc. 103 (1988), 1226-1234.

2. K. Bichteler, Stochastic integration and $L^{p}$-theory of semimartingales, Ann. Probab. 9 (1981), 49-89.

3. D. L. Burkholder Martingale transforms, Ann. Math. Statist. 37 (1966), 1494-1504.

4. __ Distribution function inequalities for martingales, Ann. Probab. 1 (1973), 19-42.

5. D. L. Burkholder and R. F. Gundy, Extrapolation and interpolation of quasi-linear operators on martingales, Acta Math. 124 (1970), 249-304.

6. R. R. Coifman and Y. Meyer, Au-delà des opérateurs pseudo-différentiels, Asterisque 57, 1978.

7. $\longrightarrow$ A simple proof of a theorem of G. David and J. L. Joruné on singular integral operators, Probability Theory and Harmonic Analysis, Marcel Dekker, 1986, pp. 61-65.

8. B. Davis, On the integrability of the martingale square function, Israel J. Math. 8 (1970), 187-190.

9. R. F. Gundy, A decomposition of $L^{1}$-bounded martingales, Ann. Math. Statist. 39 (1968), 134-138.

10. C. S. Herz, $H_{p}$-spaces of martingales, $(0<p \leq 1)$, Z. Wahrsch. Verw. Gebiete 28 (1974), 189-205.

11. D. Lepingle, Sur certains commutateurs de la théorie des martingales, Sém. Prob. 13, Lecture Notes in Math., vol. 721, Springer-Verlag, 1979, pp. 138-147.

Department of Mathematics, Cleveland State University, Cleveland, Ohio 44115

Institute of Mathematics, Academia Sinica, Beijing, China 